\title{
Broadening the detection view of high-frequency linear-array-based photoacoustic computed tomography by using planar acoustic reflectors
}

Guo Li, Jun Xia, Konstantin I. Maslov, Lihong V. Wang

Guo Li, Jun Xia, Konstantin I. Maslov, Lihong V. Wang, "Broadening the detection view of high-frequency linear-array-based photoacoustic computed tomography by using planar acoustic reflectors

," Proc. SPIE 8943, Photons Plus Ultrasound: Imaging and Sensing 2014, 89430H (3 March 2014); doi: 10.1117/12.2042725

SPIE. Event: SPIE BiOS, 2014, San Francisco, California, United States 


\title{
Broadening the detection view of high-frequency linear-array-based photoacoustic computed tomography by using planar acoustic reflectors
}

\author{
Guo Li, Jun Xia, Konstantin I. Maslov, and Lihong V. Wang* \\ Optical Imaging Laboratory, Department of Biomedical Engineering, Washington University in St. \\ Louis, One Brookings Drive, St. Louis, Missouri 63130 \\ *Corresponding author: 1hwang@biomed.wustl.edu
}

\begin{abstract}
Photoacoustic computed tomography (PACT) with a linear transducer array suffers from limited detection view. To increase the detection aperture, it is possible to circularly scan the linear transducer array around the object at the expense of imaging speed. Here we propose an alternative method to double or triple the detection view angle without sacrificing the imaging speed. By using a planar acoustic reflector which creates a virtual linear transducer array, the detection view angle is doubled. Similarly, by using two planar acoustic reflectors placed at 120 degrees to each other, we can form two virtual linear transducer arrays, and the detection view angle is tripled. This paper comparatively studies the two cases. We found that the planar acoustic reflectors greatly increase the detection aperture and thus significantly enhance the image quality of linear-array-base PACT systems.
\end{abstract}

Keywords: photoacoustic computed tomography; high frequency; linear transducer array; limited view; acoustic reflector.

\section{INTRODUCTION}

Photoacoustic tomography (PAT) forms images by detecting photoacoustic waves originating from thermal expansion caused by pulsed light absorption in biological tissues. Because acoustic scattering is several orders of magnitude less than optical scattering in biological tissue, PAT can acquire images of optical absorption at a depth beyond the optical diffusion limit, while still maintaining high spatial resolution, defined by the ultrasonic detection. ${ }^{1}$ Photoacoustic computed tomography (PACT) is one implementation of PAT, where signals acquired from multiple detection positions are reconstructed to form an image of the object. ${ }^{2}$ To accelerate data acquisition, PACT usually uses a transducer array with hundreds of elements. So far, linear transducer arrays $s^{3,4}$, circular transducer arrays ${ }^{5-7}$ and arc transducer arrays ${ }^{8}$ have all been used in PACT. Although circular transducer arrays can achieve full-view image quality, they are expensively custom-made, and their central frequencies are relatively low (smaller than $10 \mathrm{MHz}$ ), which limits the spatial resolution for small-object imaging. In contrast, linear transducer arrays are commonly used in ultrasonography. In particular, linear transducer arrays with high frequencies (central frequency $>10 \mathrm{MHz}$ ) can be used to build highresolution PACT. Moreover, linear transducer arrays can also be easily integrated with ultrasound imaging systems, leading to co-registered photoacoustic-ultrasonic imaging, which provides both optical and ultrasonic contrasts.9

However, linear-array-based PACT suffers from limited view. Due to the limited detection aperture of the whole array and the limited receiving angle of each element, linear transducer arrays cannot capture photoacoustic (PA) waves over a full 360 degrees in a two-dimensional (2-D) image plane. In fact, only boundaries that are nearly perpendicular to the acoustic axis of the linear transducer array can be detected. To increase the detection view for linear-array-based PACT, several methods have been developed. The most straightforward and easiest way is to circularly or semi-circularly scan either the linear transducer array or the imaging sample. ${ }^{10-12}$ The time-consuming scan offsets the speed advantage of using a transducer array. Gateau et al. ${ }^{13}$ proposed to use a ground glass diffuser to generate optical speckles as the illumination source, and by scanning the diffuser, they achieved full-view PACT images. However, this dynamic speckle

Photons Plus Ultrasound: Imaging and Sensing 2014, edited by Alexander A. Oraevsky, Lihong V. Wang, Proc. of SPIE Vol. 8943, 89430H - (c) 2014 SPIE · CCC code: 1605-7422/14/\$18 - doi: 10.1117/12.2042725 
method can image only shallow structures, and it still needs mechanical scanning. While an advanced algorithm may be used to improve the imaging quality by removing streaking artifacts caused by the limited view, ${ }^{14}$ it cannot recover outof-view boundaries. Ideally, we would like to enlarge the detection view of linear-array-based PACT without any mechanical scanning, in other words, without sacrificing the imaging speed. Acoustic reflectors redirect otherwise undetectable PA waves to the transducer array and hence increase the detection aperture. Cox et al. ${ }^{15}$ used two acoustic reflectors normal to the linear transducer array to get an infinitely wide virtual transducer array. However, they demonstrated this full-view imaging capability only by numerical simulations, where each transducer element was assumed to be able to receive PA waves from all directions. In reality, the limited receiving angle of each transducer element would deteriorate the theoretical predictions. Huang et al. ${ }^{16}$ experimentally proved that the detection view can be doubled by using a single 45-degree acoustic reflector adjacent to a linear transducer array. However, they utilized a lowfrequency $(5 \mathrm{MHz})$ linear transducer array, yielding low-resolution PACT images. A high-frequency linear transducer array will be more useful as it can achieve higher resolution which is essential for high image quality. As the transducer array acceptance angle is typically less than 90 degrees, the doubled detection view from a single acoustic reflector is still insufficient to image more complex features. We need to further improve the detection view of a linear-array-based PACT.

\section{EXPERIMENTAL SETUP}

In this paper, we propose high-frequency linear-array PACTs using either a detachable single 45-degree acoustic reflector or two acoustic reflectors set at 120 degrees relative to each other. They are compared against a linear-arraybased PACT without acoustic reflector, named "conventional PACT".
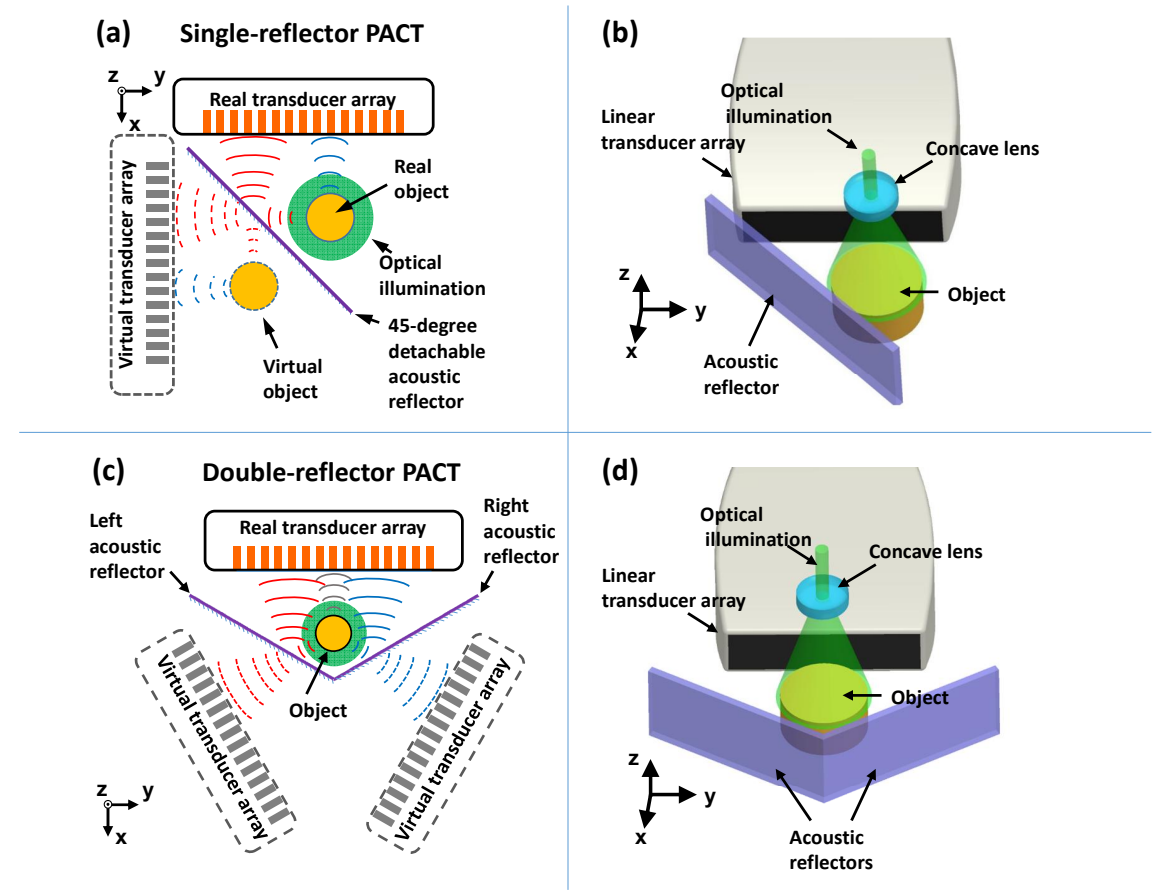

Fig. 1. Schematics of linear-array-based PACT systems using acoustic reflectors. (a), (b): single-reflector PACT; (c), (d): double-reflector PACT.

Conventional PACT can collect only PA waves propagating towards the transducer array. The acceptance angle of each element is limited by the angular spread function, which is usually less than 60 degrees for high-frequency linear transducer arrays because of the relatively large ratio of the element width to the acoustic wavelength. Therefore, in Fig. 1(a) the horizontal boundary segments with detectable PA waves propagating along the $-x$ direction can be reconstructed well, while the vertical boundary segments with undetectable PA waves propagating along the $\pm y$ direction cannot be reconstructed by the conventional PACT. To broaden the detection view, a 45-degree planar acoustic reflector was used to recover the vertical boundaries ${ }^{16}$ [Fig. 1(a)]. With the aid of the acoustic reflector, the PA waves propagating along the 
$-y$ direction are redirected to the $-x$ direction, and then received by the transducer array. While a single acoustic reflector can double the detection view, it is not enough to cover a full 360 degrees, even for large acceptance angle transducer elements. Naturally, one can add more reflectors to further extend the detection view and recover more boundaries. Fig. 1(c) shows acoustic reflectors set at an included angle of 120 degrees. Each reflector functions individually by redirecting the originally undetectable PA waves back to the transducer array elements, and together they recover more boundary features than a single reflector.

To reconstruct two-dimensional (2D) PA images acquired by all three types of linear-array-based PACTs, we used the filtered back projection algorithm. ${ }^{17}$ In contrast to the conventional PACT image reconstruction, where transducer array data are used only once, single-reflector PACT image reconstruction duplicates the data from the real transducer array to the mirrored virtual transducer array; the acoustic reflector is removed, and the filtered back projection is performed in a boundary-free infinite medium. ${ }^{18}$ Since the virtual transducer elements $\boldsymbol{r}_{\mathbf{1}}\left(x_{1}, y_{1}\right)$ are mirrored by real transducer elements $\boldsymbol{r}_{\mathbf{0}}\left(x_{0}, y_{0}\right)$ about the acoustic reflector plane, in the Cartesian coordinate they are related by

$$
\left[\begin{array}{l}
x_{1} \\
y_{1}
\end{array}\right]=\left[\begin{array}{cc}
-\cos 2 \alpha & \sin 2 \alpha \\
\sin 2 \alpha & \cos 2 \alpha
\end{array}\right]\left[\begin{array}{c}
x_{0} \\
y_{0}-y_{l}
\end{array}\right]+\left[\begin{array}{l}
0 \\
y_{l}
\end{array}\right]
$$

where $y_{l}$ is the intercept of the acoustic reflector line on the $y$ axis, and $\alpha$ is the angle between the acoustic reflection line and the linear transducer array (i.e., 45 degrees for a single reflector and 30 degrees for the left reflector in doublereflector PACT). The reconstruction of the double-reflector PACT is similar to that of a single-reflector PACT, except that there is a third transducer array which is mirrored by the real transducer array about the right reflector. This virtual transducer elements $\boldsymbol{r}_{\mathbf{1}}\left(x_{1}, y_{1}\right)$ are calculated as

$$
\left[\begin{array}{l}
x_{2} \\
y_{2}
\end{array}\right]=\left[\begin{array}{cc}
-\cos 2 \beta & -\sin 2 \beta \\
-\sin 2 \beta & \cos 2 \beta
\end{array}\right]\left[\begin{array}{c}
x_{0} \\
y_{0}-y_{r}
\end{array}\right]+\left[\begin{array}{c}
0 \\
y_{r}
\end{array}\right]
$$

where $y_{r}$ is the intercept of the right acoustic reflection line on the y axis, and $\beta$ is the angle between the acoustic reflection line and the linear transducer array (30 degrees in this case).

We experimentally tested the single-reflector PACT and double-reflector PACT using the aforementioned reconstruction algorithm. A $532 \mathrm{~nm}$ wavelength laser was used to illuminate the target from the top. The laser beam was generated from a second harmonic generator (OPOTEK Inc.) pumped by a Nd:YAG laser (Brilliant, Quantel) with $1064 \mathrm{~nm}$ output. This laser has a repetition rate of $20 \mathrm{~Hz}$ and an average pulse energy of $30 \mathrm{~mJ}$. By using an engineering diffuser, the laser beam was expanded to a diameter of $1.6 \mathrm{~cm}$ on the top surface of the target, and therefore the laser fluence was 15 $\mathrm{mJ} / \mathrm{cm}^{2}$.

For the single-reflector PACT and its corresponding conventional PACT, a high-frequency linear transducer array with a central frequency of $30 \mathrm{MHz}$ and a bandwidth of 18-28 MHz (MS400, Visualsonics Inc.) detected the PA waves. This MS400 linear transducer array has 256 elements and an effective detection aperture of $15.36 \mathrm{~mm}$. The axial resolution ( $x$-axis) was experimentally measured to be $55 \mu \mathrm{m}$ and lateral resolution (y-axis) was $117 \mu \mathrm{m}$ at a depth of $12 \mathrm{~mm}$. The hypotenuse of an optical prism (PS911, Thorlabs Inc.) was the acoustic reflector for the single-reflector PACT.

For double-reflector PACT, a high-frequency linear transducer array with a central frequency of $15 \mathrm{MHz}$ and a bandwidth of 9-18 MHz (MS200, Visualsonics Inc.) detected the PA waves. The MS200 linear transducer array has 256 elements, with a pitch of $125 \mu \mathrm{m}$ and an effective detection aperture of $32.00 \mathrm{~mm}$. The axial resolution (x-axis) in PACT was experimentally measured to be $113 \mu \mathrm{m}$, and the lateral resolution (y-axis) was $257 \mu \mathrm{m}$ at a depth of $11 \mathrm{~mm}$ in water. Glass slides were used to reflect the PA waves. The optical prism and glass slides were made of BK7 glass, which has an acoustic impedance 10 times greater than that of immersion liquid, and hence has a reflection coefficient close to unity. Although the amplitude and phase of the reflected acoustic wave change within the critical reflection angle of 26 degrees, here for simplicity we neglect this complexity and treat all reflections as total reflection without amplitude or phase change.

Both the direct PA waves and reflected PA waves were detected by either MS200 or MS400 transducer array which was connected to a commercial PA imaging system (Vevo LAZR, Visualsonics Inc.). The 256 signal channels were multiplexed to a 64-channel data acquisition system, resulting in an imaging speed of $5 \mathrm{~Hz}$. The raw channel data were then transferred to a PC where the filtered back projection algorithm was applied to reconstruct PACT images. 


\section{RESULTS}

We first imaged two simple hair phantoms as shown in Figs. 2(a) and 2(d). Straight human hairs were embedded in 3\% $\mathrm{w} / \mathrm{w}$ agar gels. The generated PA waves propagate along only certain directions, as indicated by arrows. Fig. 2(a) shows the orthogonal hair phantom with PA waves propagating along horizontal and vertical directions. Fig. 2(b) is the corresponding PA image by the conventional PACT. As expected, only the horizontal hair was recovered, and the vertical hair was missed due to the limited view. Fig. 2(c) is the corresponding PA image acquired by the single-reflector PACT, and both horizontal and vertical hairs were recovered clearly. Fig. 2(d) shows hair phantom with PA waves propagated primarily along three directions at 120 degrees relative to each other. Fig. 2(e) is the corresponding PA image acquired by the conventional PACT, which shows only the horizontal boundary. In contrast, Fig. 2(f) shows that all three hairs were recovered clearly.
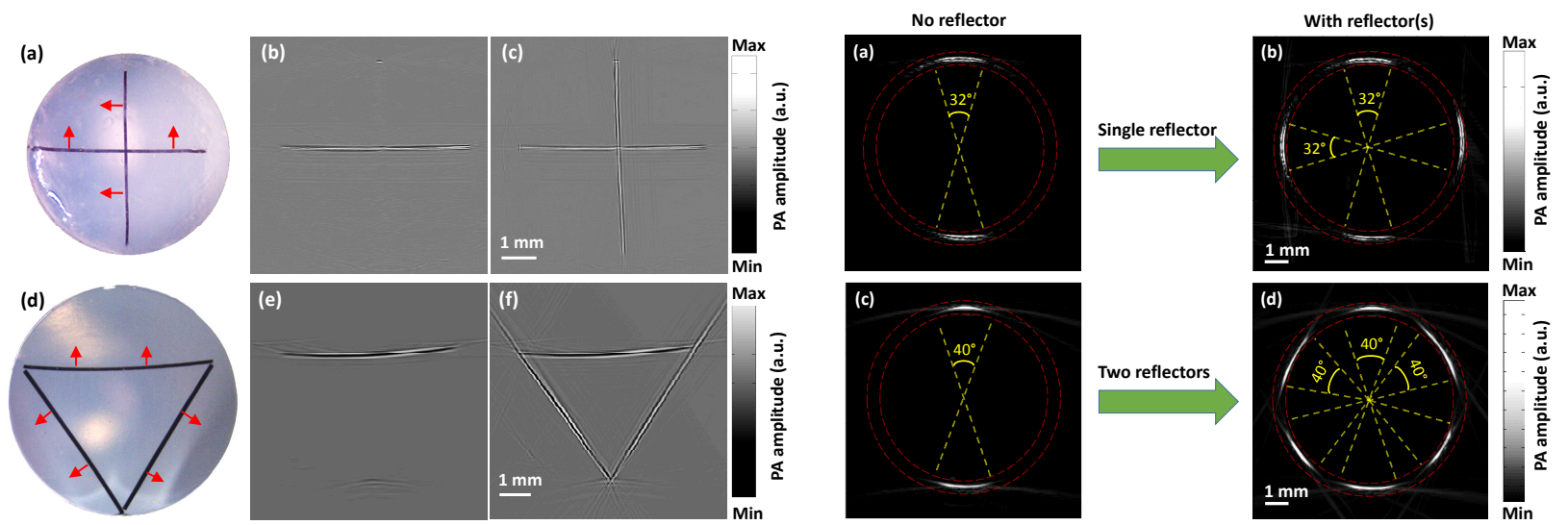

Fig. 2. Simple phantom images. (a), (d): photographs of the crossed-hair phantoms; (b), (e): reconstructed PACT images without any acoustic reflector; (c), (f): reconstructed PACT images acquired with the single reflector and two reflectors respectively.

Fig. 3. PACT images of a circular tube phantom: (a), (c) without any acoustic reflector; (b) with single planar ultrasonic reflector; (d) with two reflectors. The view angle of the linear-array-based PACT doubles and triples by using the single reflector and two acoustic reflectors respectively.

To quantitatively study the detection view enhancement, we imaged the cross-section of a tubular plastic drinking straw phantom in the $x-y$ plane. The tube had an inner-diameter of $7.3 \mathrm{~mm}$ and a wall thickness of $0.15 \mathrm{~mm}$. The circular tube generated PA waves in all 360 degrees in the 2D imaging plane, equivalent to the angular range from a point source at the center of the circle. Thereby we could quantitatively study the detection view of the three PACT systems. Fig. 3(a) shows the PA image of the circular tube acquired by the conventional PACT, where the circle center was located at 11 $\mathrm{mm}$ away from the transducer surface. Both recovered top and bottom arcs have $\sim 32$ degrees of aperture with respect to the center, and this angle indicates the receiving angle of the conventional PACT (transducer array: MS400) with respect to a point at the circle center. Here the receiving angle is limited by two factors: the azimuth angle of the entire transducer aperture and the effective receiving angle of each individual transducer element. Because the receiving angle of the MS400 transducer array element was experimentally measured to be $\sim 40$ degrees, the azimuth angle is obviously the limiting factor in this case. Fig. 3(b) shows the reconstructed circular tube phantom imaged with the single-reflector PACT system. Compared with Fig. 3(a), there are two more arcs, so the detection view of the linear-array-based PACT has increased from 64 degrees to 128 degrees, doubling the detection view of the conventional PACT system.

Fig. 3(c) shows the same circular tube phantom image acquired by the conventional PACT with the MS200 transducer array. The tube was placed at $18 \mathrm{~mm}$ away from the transducer surface, where the azimuth aperture angle is $\sim 55$ degrees so the receiving angle of transducer element limits the detection view angle in this case. As we can see from Fig. 3(c), the detection view is about 40 degrees for both top and bottom recovered arcs. Fig. 3(d) shows the tube phantom image acquired by the double-reflector PACT system. It is obvious that the detection view has been triply increased, from 80 to 240 degrees. 
To verify the detection view enhancement, we imaged complex phantoms, dehydrated skeleton leaves [Figs. 4(a) and 4(d)]. Compared with the hair phantoms in Figs. 2(a) and 2(d), the leaf phantoms have rich vascular network and more complex features. Fig. 4(b) shows the reconstructed image of leaf phantom in Fig. 4(a) with the conventional PACT. Only boundaries close to the horizontal direction were recovered. Fig. 4(c) shows the image of the phantom acquired by the single-reflector PACT, which recovered almost all boundaries of the leaf. Figs. 4(e) and 4(f) are the conventional PA image and two-reflector PACT image of the leaf phantom shown in Fig. 4(d). Similarly, we can see only some horizontal boundaries from Fig. 4(e), while Fig. 4(f) recovers most of the features identifiable in the photograph.
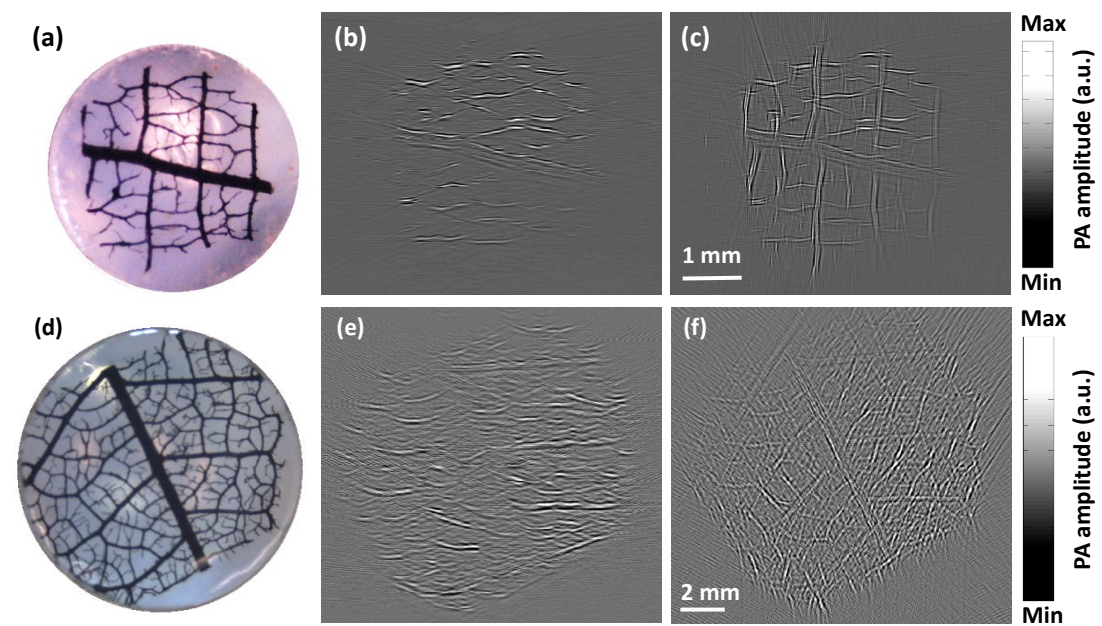

Fig. 4. Complex phantom images. (a), (d): photographs of the leaf phantoms; (b), (e): reconstructed PACT images without any acoustic reflector; (c), (f): reconstructed PACT images with the single acoustic reflector and two reflectors respectively.
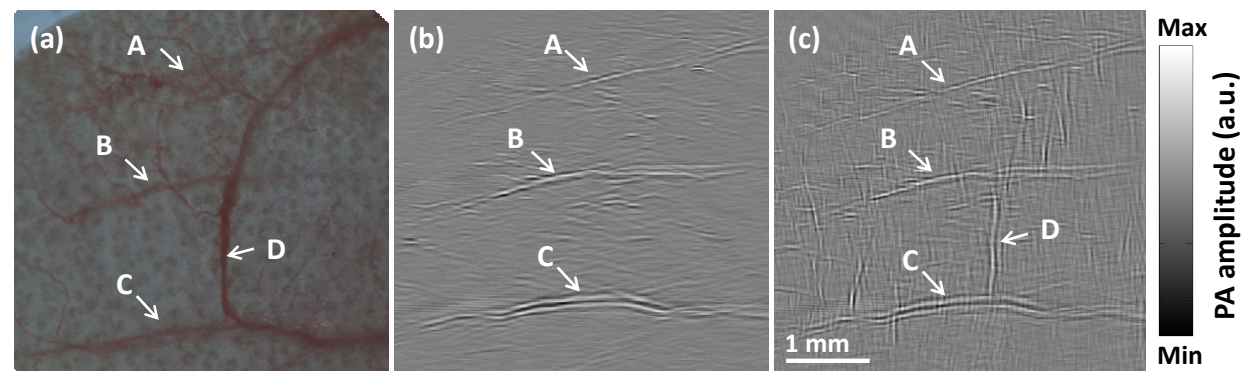

Fig. 5. In vivo images of the mouse ear. (a): photograph of the image area; (b): reconstructed PACT image without any reflector; (c): reconstructed PACT image with the single acoustic reflector.

We also acquired in vivo nude mouse ear images. During experiment, the nude mouse was anaesthetized and the mouse ear was fixed on top of a $10 \%$ gelatin phantom. The laser energy intensity was estimated to be $10 \mathrm{~mJ} / \mathrm{cm}^{2}$ on the surface of mouse ear, and was well below the ANSI limit $\left(20 \mathrm{~mJ} / \mathrm{cm}^{2}\right)$. The photograph of the mouse ear was shown in Fig. 5(a), where some big vessels are labeled. A, B, and C represent the horizontal vessels, while D represents a vertical vessel. Due to the limited view, the conventional PACT cannot recover the vessel D, as shown in Fig. 5(b). However, with the help of the 45-degree acoustic reflector, vessel D was clearly recovered, as shown in Fig. 5(c). 


\section{CONCLUSIONS}

In summary, we demonstrated three types of linear-array-based PACT systems: conventional PACT, single-reflector PACT and double-reflector PACT. With the designed configurations, single-reflector PACT and double-reflector PACT doubles and triples the original detection view of conventional PACT without sacrificing the imaging speed. The highfrequency transducer arrays used in this letter help the linear-array PACTs achieve high resolutions. Although the double-reflector PACT still has not achieved 360-degree (i.e., full-view) detection due to the limited receiving angle of the single transducer element, it is possible to achieve a full view in the future by using linear transducer arrays with larger element receiving angles and wider apertures. The fast imaging speed and large detection view of reflector PACTs make them valuable for biomedical imaging studies where both high image quality and high imaging speed are required.

\section{ACKNOWLEDGMENTS}

This work was sponsored by National Institute of Health (NIH) grants DP1 EB016986 (NIH Director's Pioneer Award), R01 CA186567 (NIH Director's Transformative Research Award), R01 EB016963, R01 CA134539, R01 EB010049, R01 CA159959 and 1S10RR026922-01. L.W. has a financial interest in Microphotoacoustics, Inc. and Endra, Inc., which, however, did not support this work. K.M. has a financial interest in Microphotoacoustics, Inc., which did not support this work.

\section{REFERENCES}

[1] L. V. Wang, Nat Photon 3 (9), 503-509 (2009).

[2] L. V. Wang and S. Hu, Science 335 (6075), 1458-1462 (2012).

[3] L. Song, K. Maslov, K. K. Shung and L. H. Wang, J. Biomed. Opt. 15 (2) (2010).

[4] C. Kim, T. N. Erpelding, K. Maslov, L. Jankovic, W. J. Akers, L. Song, S. Achilefu, J. A. Margenthaler, M. D. Pashley and L. V. Wang, J. Biomed. Opt. 15 (4), 046010-046010 (2010).

[5] J. Gamelin, A. Maurudis, A. Aguirre, F. Huang, P. Guo, L. V. Wang and Q. Zhu, Opt. Express 17 (13), 1048910498 (2009).

[6] J. Xia, Z. Guo, K. Maslov, A. Aguirre, Q. Zhu, C. Percival, and L. V. Wang, J. Biomed. Opt. 16 (9), 090505 (2011).

[7] J. Xia, M. R. Chatni, K. Maslov, Z. Guo, K. Wang, M. Anastasio and L. V. Wang, J. Biomed. Opt. 17 (5), 050506050501 (2012).

[8] H.-P. Brecht, R. Su, M. Fronheiser, S. A. Ermilov, A. Conjusteau and A. A. Oraevsky, J. Biomed. Opt. 14 (6), 064007-064007 (2009).

[9] J. Yuan, G. Xu, Y. Yu, Y. Zhou, P. L. Carson, X. Wang and X. Liu, J. Biomed. Opt. 18 (8), 086001-086001 (2013).

[10] D. W. Yang, D. Xing, S. H. Yang and L. Z. Xiang, Optics Express 15 (23), 15566-15575 (2007).

[11] R. A. Kruger, J. W. L. Kiser, D. R. Reinecke and G. A. Kruger, Medical Physics 30 (5), 856-860 (2003).

[12] J. Gateau, M. A. A. Caballero, A. Dima and V. Ntziachristos, Medical Physics 40 (1), 013302-013311 (2013).

[13] J. Gateau, T. Chaigne, O. Katz, S. Gigan and E. Bossy, Optics Letters 38 (23), 5188-5191 (2013).

[14] S. Ma, S. Yang and H. Guo, Journal of Applied Physics 106 (12), - (2009).

[15] B. T. Cox, S. R. Arridge and P. C. Beard, Inverse Problems 23 (6), S95 (2007).

[16] B. Huang, J. Xia, K. Maslov and L. V. Wang, J. Biomed. Opt. 18 (11), 110505-110505 (2013).

[17] M. Xu and L. V. Wang, Physical Review E 71 (1), 016706 (2005).

[18] L. V. Wang and X. Yang, J. Biomed. Opt. 12 (1), 014027-014027 (2007). 